\title{
The Influence of Detergents on the Bleaching Process, Laser Properties of Rhodamine 6G and B in Aqueous Solution
}

\author{
Z. Konefał \\ Institute of Physics, University of Gdańsk, 80-952 Gdańsk, Poland \\ Z. Naturforsch. 34a, 551-556 (1979); received Dezember 5, 1977
}

\begin{abstract}
The bleaching and the laser properties of Rhodamine $6 \mathrm{G}$ and $\mathrm{B}$ in aqueous detergent solutions have been studied. The rate of bleaching depends on the concentration of the detergent. The relative quantum yields of the bleaching process for these solutions have been determined. It is found that the influence of detergents on the generation efficiency depends on the type of dye employed. From the results obtained it is concluded that the detergents influence the population of the triplet state of the lasing dye.
\end{abstract}

\section{Introduction}

Detergents bear potentialities for laser techniques; for instance, they prevent dimerization, they allow for a dissolution of non soluble dyes in water [1] and they influence the triplet state of the lasing dye and the bleaching processes. A detergent in an aqueous dye solutions may significantly increase the efficiency of the generation process $[2,3]$.

Bleaching processes in solutions have already been investigated in a series of papers $[4-10]$. The present work deals with the bleaching of Rhodamine $6 \mathrm{G}$ and $\mathrm{B}$ in aqueous solutions containing various quantities of the following detergents:

\section{Hostapon $\quad \mathrm{C}_{12} \mathrm{H}_{25} \mathrm{SO}_{4} \mathrm{Na}$}

2. Sulfapol $\quad \mathrm{C}_{12} \mathrm{H}_{25}-\mathrm{C}_{6} \mathrm{H}_{4}-\mathrm{SO}_{3} \mathrm{Na}$

3. Rokafenol N-8 p- $\mathrm{C}_{9} \mathrm{H}_{19}-\mathrm{CH}_{4}-\mathrm{O} / \mathrm{OCH}_{2} \mathrm{CH}_{2} / 8 \mathrm{H}$

4. Rokafenol 0-22 p- $\mathrm{C}_{8} \mathrm{H}_{17}-\mathrm{C}_{6} \mathrm{H}_{4}-\mathrm{O} / \mathrm{OCH}_{2} \mathrm{CH}_{2} /{ }_{22} \mathrm{H}$

5. Triton X-100 $\mathrm{C}_{8} \mathrm{H}_{17}-\mathrm{C}_{6} \mathrm{H}_{4}-\mathrm{O} / \mathrm{OCH}_{2} \mathrm{CH}_{2} /{ }_{10} \mathrm{H}$

6. Tween 80

$$
\begin{aligned}
& \mathrm{CH}_{3} / \mathrm{CH}_{2} /{ }_{7} \mathrm{CH} \\
& =\mathrm{CH} / \mathrm{CH}_{2} /{ }_{7}-\mathrm{COOCH}_{2} \mathrm{CH}-\mathrm{CH}-\mathrm{CH}-\mathrm{CH} \\
& \\
& \quad \text { OH OH OH OH } \\
& -/ \mathrm{OCH}_{2} \mathrm{OCH}_{2} \mathrm{CH}_{2} / 40 \mathrm{H}
\end{aligned}
$$

The bleaching processes were investigated by measuring changes in the absorption spectrum and in the fluorescence quantum yield caused by strong illumination. Both measurements were made using a Zeiss-Jena spectrometer in conjunction with an apparatus described earlier [18].

Reprint requests to Dr. Z. Konefal. Please order a reprint rather than making your own copy.

$0340-4811 / 79 / 0500-0551 \$ 01.00 / 0$
Changes of the triplet state parameters caused by the detergents were also measured and analysed on the basis of simplified solutions of the kinetic equations for a dye laser.

\section{Experimental}

During strong illumination of the dye solution certain types of photoreactions take place which cause the fluorescence quantum yield to decrease and the absorption spectrum to change. Figure 1 shows the absorption spectrum obtained on illumination with two air flash lamps of an aqueous solution of Rhodamine $6 \mathrm{G}$ without a detergent. With increasing number of flashes the intensity of the first absorption band, i.e. the dye concentration distinctly decreases. Simultaneously the curve becomes broader. In the short wavelength region a rise of the absorption occurs due to the photoreaction products. In the long wavelength region the absorption by photoreaction products is smaller but due to the overlap with the emission spectrum there is a serious loss of light generation.

Figure 2 shows the long wavelength absorption band of Rhodamine $6 \mathrm{G}$ in aqueous solution with various concentrations of Rokafenol 0-22. At the small detergent concentration the sensitivity to light is increased.

Figure 3 shows the fluorescence intensity and quantum yield as a function of the number of flashes. It is noticeable that the stability of the solutions increases up to detergent concentrations of $10^{-1} \mathrm{M}$. Further addition of detergent generally leads to insignificant changes.

It was of practical interest to investigate which detergent most influenced the population of the triplet state. 


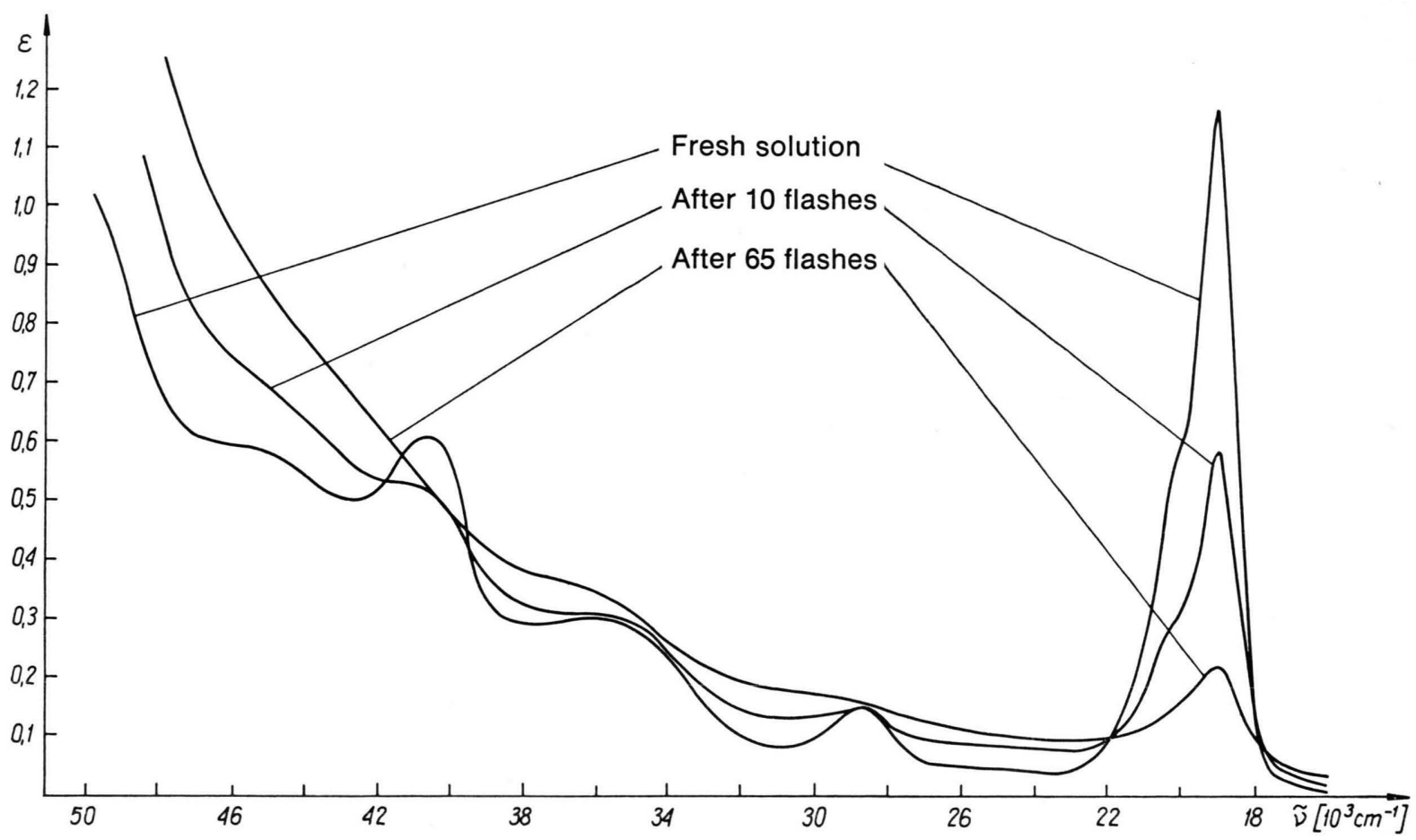

Fig. 1. Absorption spectrum of Rhodamine $6 \mathrm{G}$ aqueous solution (without detergent). Parameter: number of lampflashes. Dye concentration $c=2 \cdot 10^{-5} \mathrm{M}$.
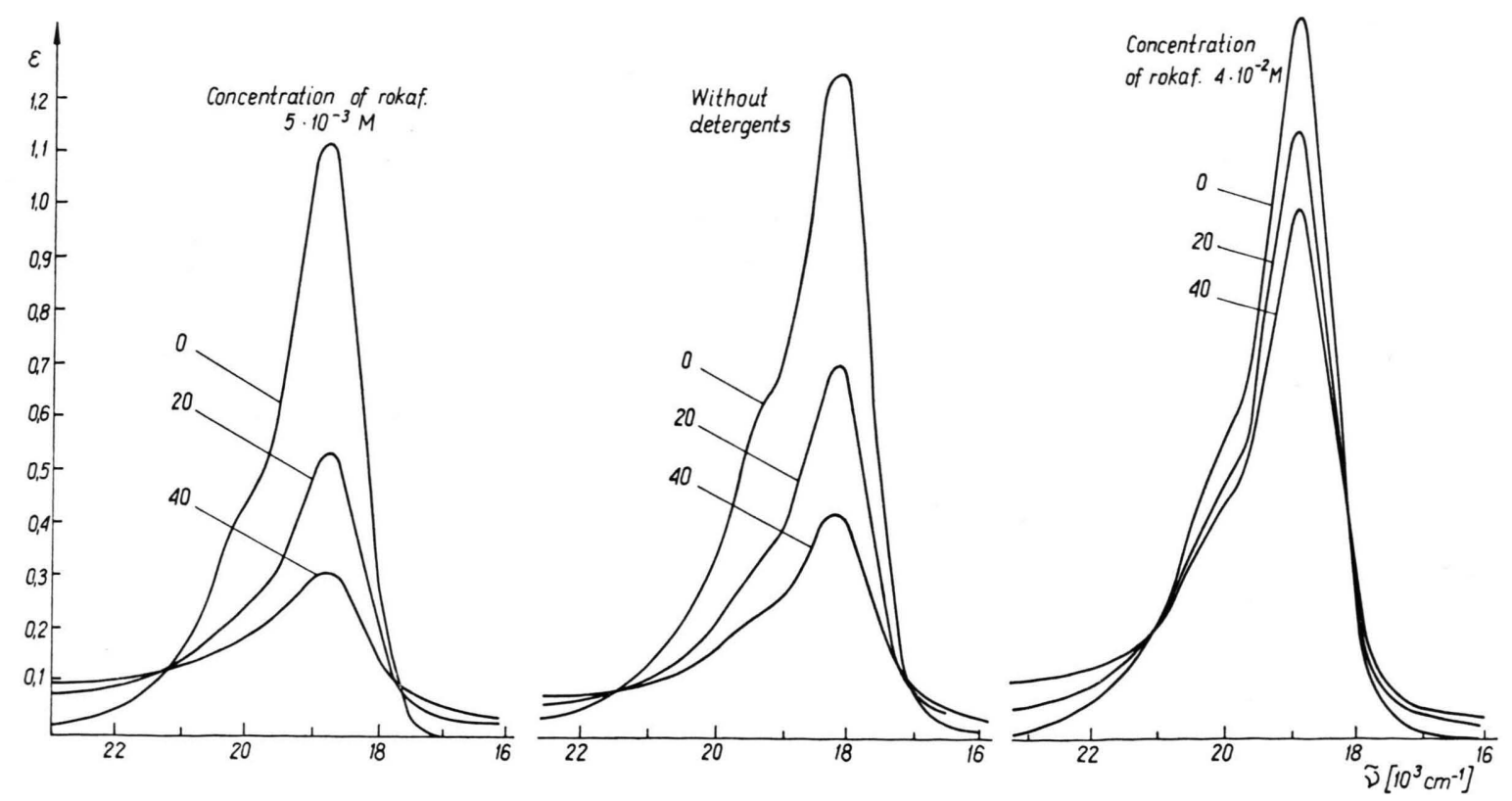

Fig. 2. Absorption spectra of Rhodamine $6 \mathrm{G}$ water solutions with additive Rokafenol 0-22 versus optical wavenumber. The number of flashes is indicated at the curves. 



$2 \cdot 10^{-5} \mathrm{M}$ Rhodamine $6 G+$ rokafenol $0-22$
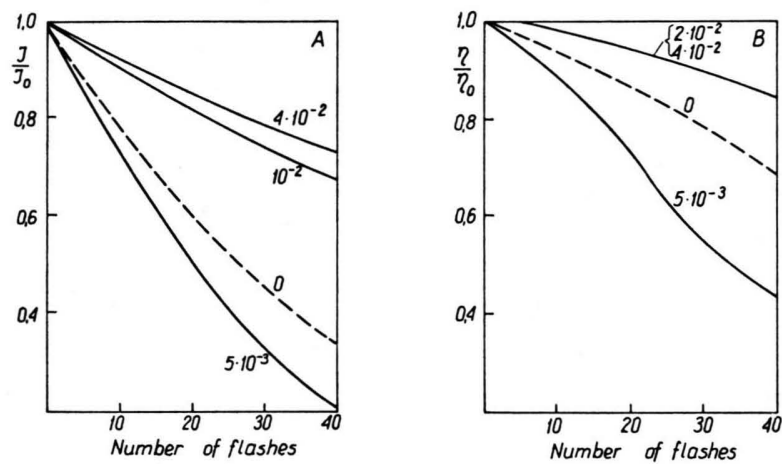

Fig. 3. Relative values of fluorescence intensity $(A)$ and fluorescence quantum yield $(B)$ versus the number of lampflashes. Parameter: Detergent concentration. Dashed lines refer to solutions without detergent.

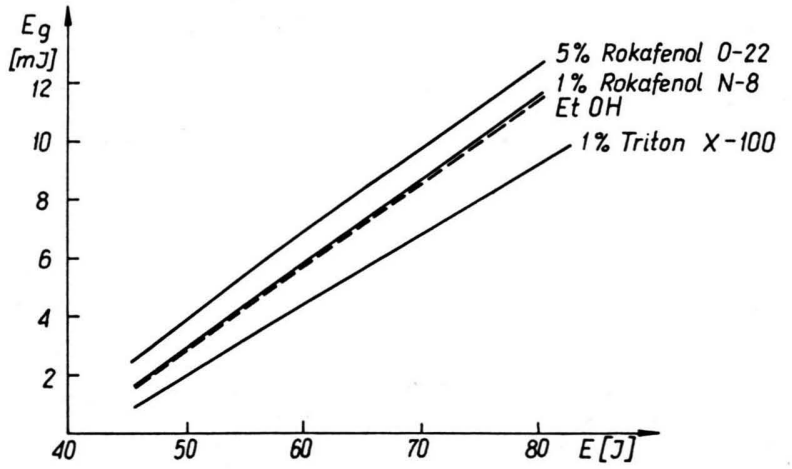

Fig. 4. The generation energy of rhodamine $6 \mathrm{G}$ aqueous solution versus the capacitor energy for various detergents.

Figure 4 and Fig. 5 show detailed results for the generation energy versus the pumping energy for Rhodamine $6 \mathrm{G}$ and Rhoramine $\mathrm{B}$. As can be seen, there is an appreciable difference in the performance of detergents.
Fig. 5. The generation energy of Rhodamine B aqueous solution with various detergents versus capacitor energy.

\section{Discussion}

The absorption coefficient in the long wavelength band and the fluorescence quantum yield both decrease with increasing intensity of illumination. This is due to photochemical processes which reduce the number of active molecules and give rise to the formation of light absorbing and nonfluorescent molecules. One may assume that it is the concentration increase of the latter molecules which causes the decrease in the quantum yield. If this is so, one can determine the relationship between the concentration of active molecules in the solution and the number of lamp flashes. The fluorescence quantum yield of the solution is given by:

$$
\eta=\eta_{0} \frac{n \sigma_{\mathrm{a}}}{n \sigma_{\mathrm{a}}+\sum_{W=1}^{P} n_{W} \sigma_{W}}
$$

where $\eta_{0}$ is the yield of the fresh solution, $n$ the dye concentration per unit volume, $\sigma_{\mathrm{a}}$ the absorption cross-section of the active molecules, $n_{W}$ the concentration of photoreaction products $(W=1,2$, $3, \ldots, p)$, and $\sigma_{W}$ the absorption cross-section of the photoreaction products.

The rate of absorption is

$$
\varepsilon=-\log \left(I / I_{d=0}\right),
$$

where $d$ is the thickness of the solution layer. Evidently

$$
\varepsilon=(\log e)\left(n \sigma_{\mathrm{a}}+\sum_{W=1}^{P} n_{W} \sigma_{W}\right) d
$$

and

$$
\varepsilon_{0}=(\log e) n_{0} \sigma_{\mathrm{a}} d,
$$


where $n_{0}$ is the initial concentration of active molecules. If $\eta / \eta_{0}$ and $\varepsilon / \varepsilon_{0}$ are measured, the ratio $n / n_{0}$ is obtained from

$$
\frac{n}{n_{0}}=\frac{\varepsilon}{\varepsilon_{0}} \frac{\eta}{\eta_{0}} .
$$

As a first approximation we can assume that the bleaching process is described by the equation

$$
\mathrm{d} n / \mathrm{d}(N \tau)=-\Phi n \int_{\nu} \sigma_{\mathrm{a}}(v) F(v) \mathrm{d} v,
$$

where $\Phi$ is the quantum yield of the bleaching process, $F(v)$ the pumping light intensity of the flash lamp in photons $\mathrm{cm}^{-2} \mathrm{~s}^{-1}, N$ the number of flashes and $\tau$ the flash duration. Integration of (6) leads to

$$
\ln \left(n / n_{0}\right)=K N,
$$

with

$$
K=-\Phi \tau \int_{v} \sigma_{\mathrm{a}}(v) F^{\prime}(v) \mathrm{d} v .
$$

For concentrations considerably exceeding the appropriate critical level our experimental results show a constant slope $K$ of the plots of $\ln \left(n / n_{0}\right)$ versus $N$.

We may assume as a first approximation that

$$
\int_{\nu} \sigma_{\mathrm{a}}(v, c) F(v) \mathrm{d} v
$$

is independent of the detergent's presence in solution*. One then has

$$
\frac{K_{\mathrm{d}}(c)}{K(0)}=\frac{\Phi_{\mathrm{d}}(c)}{\Phi(0)}
$$

where $K(0)$ is the slope for the solution without detergent and $K_{\mathrm{d}}(c)$ the slope for the solution with the detergent concentration $c . \Phi_{\mathrm{d}}(c) / \Phi(0)$ values are given in Tables 1 and 2.

Bleaching of Rhodamine $6 \mathrm{G}$ is best prevented by Rokafenol N-8 and 0-22. These two detergents lower the yield of the bleaching process by nearly a factor of three. For Rhodamine B, however, Triton X-100 is best in this respect. It reduces the bleaching to one quarter that of the pure solution.

At detergent concentrations lower than the critical one, that is the lowest concentration at which

* For other kinds of detergents one can observe shifts in the absorption spectrum. However the integral absorption is approximately constant.

The intensity distribution for a flash-lamp in the area of the dye absorption curve is a slowly changing function of the frequency. Taking this into account our assumption is justified.
Table I. The $K$ and $\Phi_{\mathrm{d}}(c) / \Phi(0)$ values of Rhodamine $6 \mathrm{G}$ $\left(2 \cdot 10^{-5} \mathrm{M}\right)$ aqueous solutions for different kinds of detergents.

\begin{tabular}{lllll}
\hline Pos. & Detergent & $\begin{array}{l}\text { Concen- } \\
\text { tration }\end{array}$ & $K$ & $\Phi_{\mathrm{d}}(c) / \Phi(0)$ \\
\hline $\mathbf{1}$ & Without detergent & - & 0.81 & $\mathbf{1 . 0}$ \\
$\mathbf{2}$ & Tween-80 & $\mathbf{5 \%}$ & 0.60 & 0.75 \\
3 & Hostapon & $\mathbf{5 \%}$ & 0.41 & 0.50 \\
$\mathbf{4}$ & Triton X-100 & $\mathbf{5 \%}$ & $\mathbf{0 . 3 4}$ & 0.40 \\
5 & Rokafenol 0-22 & $\mathbf{5 \%}$ & $\mathbf{0 . 3 1}$ & 0.37 \\
$\mathbf{6}$ & Rokafenol N-8 & $\mathbf{5 \%}$ & $\mathbf{0 . 2 6}$ & $\mathbf{0 . 3 1}$ \\
\hline
\end{tabular}

Table II. The $K$ and $\Phi_{\mathrm{d}}(c) / \Phi(0)$ values of Rhodamine B $\left(2 \cdot 10^{-5} \mathrm{M}\right)$ aqueous solutions for different kinds of detergents.

\begin{tabular}{lllll}
\hline Pos. & Detergent & $\begin{array}{l}\text { Concen- } \\
\text { tration }\end{array}$ & $K$ & $\Phi_{\mathrm{d}}(c) / \Phi(0)$ \\
\hline 1 & Without detergent & - & 1.1 & 1.0 \\
2 & Tween-80 & $5 \%$ & 0.63 & 0,57 \\
3 & Hostapon & $5 \cdot 10^{-2} \mathrm{M}$ & 0.83 & 0.75 \\
4 & Rokafenol N-8 & $5 \cdot 10^{-2} \mathrm{M}$ & 0.62 & 0.56 \\
5 & Rokafenol O-22 & $5 \cdot 10^{-2} \mathrm{M}$ & 0.60 & 0.54 \\
6 & Triton X-100 & $5 \%$ & 0.27 & 0.24 \\
\hline
\end{tabular}

micelles form, a reduced resistance to the bleaching phenomenon is observed. Presumably this is due to the presence of free detergent molecules, i.e. to molecules that do not form micelles (ions) of the detergent. Compared with molecules forming micelles, free molecules are more inclined to form associates with a dye. When a detergent concentration is higher than the critical one, the resistance of the solution to bleaching is larger. The resistance to bleaching reaches saturation at concentrations in the range of $5 \cdot 10^{-2}-1 \cdot 10^{-1} \mathrm{M}$.

The micellous structure of the solution is well formed at these concentrations [11-13]. It is also known that dye molecules do not occupy random positions in the solution but form a local structure. In these circumstances the photoreaction processes may be stopped either for energetic reasons or because there is a strong limitation on molecular mobility. There may be other factors such as the UV absorption of the detergent which increase the photochemical stability of the solutions at high detergent concentrations.

In the papers $[10,14,15]$ the greater stability against bleaching is ascribed to quenching of the triplet state of the dye molecules by the quenchers.

The results given earlier concerning the influence of the pumping energy on the generated energy 
$a$.

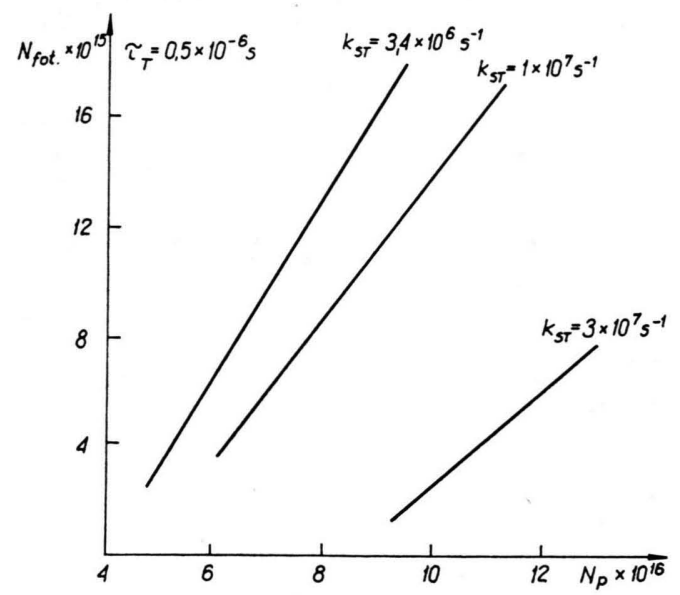

b.

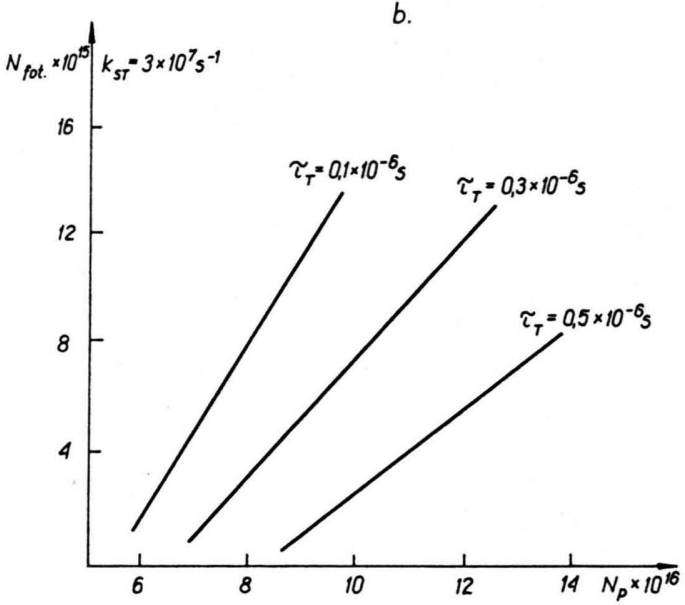

Fig. 6. The generation energy versus pumping energy calculated with a) $\tau_{\mathrm{T}}=0.5 \cdot 10^{-6} \mathrm{~s}$ and $k_{\mathrm{ST}}$ as free parameter; b) $k_{\mathrm{ST}}=3 \cdot 10^{7} \mathrm{~s}^{-1}$ and $\tau_{\mathrm{T}}$ as the free parameter.

have shown different efficiencies for various detergent solutions. Also, the efficiency of the detergent solution depends on the type of lasing dye molecules. For instance, Rokafenol $\mathrm{O}-22$ raises the generating efficiency particularly for Rhodamine $6 \mathrm{G}$ whereas for Rhodamine $\mathrm{B}$ the best detergent is Triton X-100.

Since for our materials the luminescence parameters are approximately equal and dye dimerization does not occur, the differences in the efficiencies of generation are probably caused by the dependence of the triplet state population on the detergent type. The efficiency of conversion of excitation energy into generation energy depends on the population of the triplet state.

The slope of the curve of generation energy against pumping energy is proportional to the efficiency of the dye solution. The population of the triplet state depends on the two parameters defining the transition probabilities $S_{1}-T_{1}\left(k_{S T}\right)$ and $T_{1}-S_{0}\left(\tau_{T}^{-1}\right)$. In this analysis the $\tau_{T}$ and $k_{S T}$ values are treated as parameters. The dependence of generation energy versus pumping energy can be analysed on the basis of rate equations describing the generation process [16-18]. Since the duration of the excitation pulse is in the range of microseconds and both the lifetime of photons in the cavity and the lifetime of the $S_{1}$ state are in the range of nanoseconds, it was assumed that the number of emitted photons and the population of the $S_{1}$ state are in quasistatic equilibrium during the exciting pulses. Since the $\tau_{T}$ and $k_{S T}$ values are comparable to the excitation duration time, this assumption is not valid for the triplet state. Therefore we have calculated the generation efficiencies for different $k_{S T}, \tau_{T}$ and for different pumping energies. These calculations were performed on a MERA 303 minicomputer. The time distribution of the intensity of the pumping lamp was taken as the pumping energy and the experimentally determinated luminescence parameters of Rhodamine $6 \mathrm{G}$ in aqueous solutions were used as input data (Figures 6a, 6b).

The constant $k_{S T}$ was determined from the measured quantum yield and the fluorescence lifetime of the Rhodamine $6 \mathrm{G}$ solution [3] assuming that the transition probability of radiationless transitions $S_{1}-S_{0}$ was negligible.

By comparing the experimental (Figs. 4, 5) and calculated (Figs. 6a, 6 b) slopes of the generation energy versus pumping energy for the Rhodamine $6 \mathrm{G}$ solution one can estimate the influence of the detergent on $k_{S T}$ and $\tau_{T}$. Assuming that detergents do not affect the $k_{S T}$ parameter (we assumed that $k_{S T}=3 \cdot 10^{7} \mathrm{~s}^{-1}$ ) it is evident that one can compare Rokafenol $\mathrm{O}-22$ in aqueous solution with Triton X-100.

In the above discussion only two extreme cases have been considered.

In reality the detergent probably effects both parameters $\tau_{T}$ and $k_{S T}$ simultaneously. 


\section{Summary}

We have shown that the bleaching rate of the solution depends on the concentration of the detergent. Solutions with a detergent concentration below or little above a critical value can be easyly bleached. It may be supposed that the interaction of a dye molecule with free detergent molecules lowers its stability. Above the critical concentration the resistance of the solution to bleaching begins to rise.

The reduction of bleaching reaches saturation for detergent concentration of $5 \cdot 10^{-2} \mathrm{M}$ to $1 \cdot 10^{-1} \mathrm{M}$. The relative quantum yield of the bleaching process for Rhodamine $6 \mathrm{G}$ and $\mathrm{B}$ solutions containing detergents has been determined. The best detergents for Rhodamine $6 \mathrm{G}$ appears to be Rokafenol N-8 and O-22. They decrease the rate of bleaching by a factor of three. The best detergent for Rhodamine $\mathrm{B}$ appears to be Triton-100 which causes a four fold decrease.

The results of our measurements of generated light energy versus pumping energy prove that the same dye solutions with different types of detergent show differing degrees of light generation efficiency. It also appears that the influence of the detergent on the generation efficiency depends on the type of leasing dye molecule. The best detergent for

[1] S. A. Tuccio, K. H. Drexhage, and G. A. Reynolds, Opt. Comm. 7, 248 (1973).

[2] G. A. Kenney-Wallace, I. H. Flint, and S. G. Wallace, Chem. Phys. Lett. 32, 71 (1975).

[3] Z. Konefal, E. Lisicki, and T. Marszałek, Acta Physica Polonica A 52, 1 (1977).

[4] I. Weler, Phys. Letters 37 A, 179 (1971).

[5] D. Kato and A Sugimura, Opt. Comm. 10, 327 (1974).

[6] I. Weber, Opt. Comm. 7, 420 (1973).

[7] N. A. Rubinow, T. J. Smolskaja, and S. S. Michnow, Žurn. Prikł. Spektr. 13, 368 (1970).

[8] B. H. Winters, H. L. Mandelberg, and W. B. Mohr, Appl. Phys. Lett. 25, 723 (1973)

[9] E. J. Schimitschek, I. A. Trias, P. R. Hammond, and R. L. Atkina, Opt. Comm. 11, 352 (1974).

[10] M. Yamashita and H. Kashiwagi, IEEE I. of Quantum Electronics QE-12, 90 (1976).
Rhodamine $6 \mathrm{G}$ aqueous solution appears to be Rokafenol O-22, which however, when added to Rhodamine B solution results in the lowest efficiency. The best detergent for Rhodamine B solution is Triton $\mathrm{X}-100$. The reason why the same dye has different generation efficiencies for various detergents may be the differing influence of detergent molecules on the population of the triplet state. Such a conclusion is plausible since the remaining parameters do not depend on the type of detergent used.

The influence of the parameters $k_{S T}$ and $\tau_{T}$ on the generated light energy has been analysed on the basis of an approximate solution of the kinetic equations for a dye laser. By a comparison of the theoretically calculated generation energy for different pumping energies with the corresponding experimental data we have estimated the changes of $k_{S T}$ and $\tau_{T}$ caused by various detergents.

\section{Acknowledgement}

I wish to thank Doc. Dr. hab. T. Marszałek for his hospitality during my stay at N. Copernicus University in Torun and for stimulating talks. Also, I which to thank Doc. Dr. hab. J. Heldt for his assistance in completing this paper for publication.

[11] M. J. Schick, Nonionic Surfactants, M. Dekker Inc., New York 1967.

[12] L. M. Kushner and W. D. Hubburd, J. Phys. Chem. 58, 1163 (1974)

[13] S. Yedgar, Y. Barenholz, and G. V. Cooper, Biochim. Biophys. Acta 363, 98 (1974).

[14] A. Tuccio and F. C. Strome, Appl. Opt. 11, 64 (1972).

[15] D. W. Phillion, D. I. Kurzenga, and A. E. Siegman, J. Chem. Phys. 61, 3828 (1974).

[16] D. P. Sorakin, I. R. Lankard, V. L. Moruzzi, and E. C. Hammond, J. Chem. Phys. 48, 4726 (1968).

[17] A. Pappalardo, H. Samelson, and A. Lempicki, J. Appl. Phys. 43, 3776 (1972).

[18] T. Marszałek, Praca habilitacyjna, University of Toruń, Toruń 1974. 